OPEN ACCESS

Edited by:

Hui Shen,

Tongji University, China

Reviewed by:

Idessania Costa

State University of Londrina, Brazil Masoomeh Shams-Ghahfarokhi,

Tarbiat Modares University, Iran Mao-Mao An,

Shanghai Tenth People's Hospital,

China

*Correspondence.

Kang Zeng

nfpfkzk@126.com

Xiao-wen Huang

hxw617@163.com

†These authors have contributed equally to this work

Specialty section:

This article was submitted to Infectious Agents and Disease,

a section of the journal

Frontiers in Microbiology

Received: 28 August 2021 Accepted: 11 November 2021 Published: 10 December 2021

Citation:

Xu M-n, Li L, Pan W, Zheng $\mathrm{H}-\mathrm{x}$, Wang $M-l$, Peng X-m, Dai S-q, Tang $Y-m$, Zeng $K$ and Huang $X-w$ (2021) Zinc Oxide Nanoparticles Prime a Protective Immune Response in Galleria mellonella to Defend Against Candida albicans.

Front. Microbiol. 12:766138. doi: 10.3389/fmicb.2021.766138

\section{Zinc Oxide Nanoparticles Prime a Protective Immune Response in Galleria mellonella to Defend Against Candida albicans}

\author{
Mei-nian Xu't, Li Li'1', Wen Pan², Huan-xin Zheng', Meng-lei Wang', Xiao-ming Peng', \\ Si-qi Dai', Ying-mei Tang ${ }^{1}$, Kang Zeng ${ }^{1 *}$ and Xiao-wen Huang ${ }^{1 *}$
}

${ }^{1}$ Department of Dermatology, Nanfang Hospital, Southern Medical University, Guangzhou, China, ${ }^{2}$ Division of Infectious
Diseases, Rhode Island Hospital, Warren Alpert Medical School of Brown University, Providence, Rl, United States

Purpose: Zinc oxide nanoparticles (ZnO-NPs) have exerted antimicrobial properties. However, there is insufficient evaluation regarding the in vivo antifungal activity of $\mathrm{ZnO}$ NPs. This study aimed to investigate the efficacy and mechanism of ZnO-NPs in controlling Candida albicans in the invertebrate Galleria mellonella.

Methods: Galleria mellonella larvae were injected with different doses of ZnO-NPs to determine their in vivo toxicity. Non-toxic doses of ZnO-NPs were chosen for prophylactic injection in G. mellonella followed by C. albicans infection. Then the direct in vitro antifungal effect of $\mathrm{ZnO}-\mathrm{NPs}$ against $C$. albicans was evaluated. In addition, the mode of action of ZnO-NPs was assessed in larvae through different assays: quantification of hemocyte density, morphology observation of hemocytes, characterization of hemocyte aggregation and phagocytosis, and measurement of hemolymph phenoloxidase (PO) activity.

Results: Zinc oxide nanoparticles were non-toxic to the larvae at relatively low concentrations ( $\leq 20 \mathrm{mg} / \mathrm{kg}$ ). ZnO-NP pretreatment significantly prolonged the survival of $C$. albicans-infected larvae and decreased the fungal dissemination and burden in the C. albicans-infected larvae. This observation was more related to the activation of host defense rather than their fungicidal capacities. Specifically, ZnO-NP treatment increased hemocyte density, promoted hemocyte aggregation, enhanced hemocyte phagocytosis, and activated $\mathrm{PO}$ activity in larvae.

Conclusion: Prophylactic treatment with lower concentrations of ZnO-NPs protects G. mellonella from $\mathrm{C}$. albicans infection. The innate immune response primed by $\mathrm{ZnO}$ NPs may be part of the reason for the protective effects. This study provides new evidence of the capacity of ZnO-NPs in enhancing host immunity and predicts that ZnO-NPs will be attractive for further anti-infection applications.

Keywords: zinc oxide nanoparticles, Galleria mellonella, Candia albicans, innate immunity, anti-infection 


\section{INTRODUCTION}

Candida albicans is a prevalent opportunistic pathogen that can cause mucosal, cutaneous, and deep-seated organ infections in humans. C. albicans infection is the leading cause of systemic candidiasis, which is responsible for the high mortality among immunosuppressed or immunocompromised patients in hospitals (Gudlaugsson et al., 2003; Kullberg and Arendrup, 2016). Due to the limited antifungals against C. albicans and the continuous emergence of drug resistance, more effective and long-term antifungal materials are desperately needed (Salazar et al., 2020).

In recent years, metallic nanoparticles (NPs), such as silver, gold, copper, zinc, and iron NPs, have exhibited antimicrobial potentials (Staron and Dlugosz, 2021). NPs exert antimicrobial properties through multifaceted mechanisms, including, but not limited to, induction of reactive oxygen species (ROS), cell membrane damage, and interaction with biomolecules (Kim et al., 2009; Staron and Dlugosz, 2021). Among diverse types of NPs, silver nanoparticles (Ag-NPs) are most widely used as antimicrobials. However, the application of Ag-NPs has drawn public concerns due to the side effects, including toxicity, oxidative stress, and inflammatory response (Burns et al., 2021). Different from Ag-NPs, zinc oxide nanoparticles (ZnO-NPs) are safer to mammalian cells (Bondarenko et al., 2013; Efthimiou et al., 2021). Bondarenko et al. (2013) pointed out that the median lethal doses (LD50) of Ag-NPs and ZnONPs for mammalian cells were 11.3 and $43 \mathrm{mg} / \mathrm{L}$, respectively. Moreover, ZnO-NPs possess several other advantages, such as chemical and thermal stability, high oxidation energy, and good antimicrobial properties, facilitating their wide application (Sharma et al., 2020). ZnO-NPs have a wide range of antifungal effects, including C. albicans, Trichophyton, Microsporum canis, mentagrophytes, Aspergillus flavus, Sclerotinia homoeocarpa, and Fusarium oxysporum (Sun et al., 2018). In addition, $\mathrm{ZnO}-\mathrm{NPs}$ can increase the antifungal capacity of other materials, such as flax pulp (Abo Elgat et al., 2020). These studies suggest that $\mathrm{ZnO}$-NPs have the potential to be an alternative to the classical antifungals. However, the current research mainly focused on the potential applications of NPs in agriculture (Hernandez-Melendez et al., 2018). Most antimicrobial data of $\mathrm{ZnO}-\mathrm{NPs}$ were obtained from in vitro assays, stimulating the in vivo studies to elucidate their toxicity and antifungal activity.

Considering their specific characteristics, the invertebrate Galleria mellonella larvae have been widely used as an animal model for evaluating the virulence of pathogens and the efficacy of antimicrobial agents. For instance, larvae have a short lifespan, low cost, no ethical implications, and no requirement for special lab equipment (Cutuli et al., 2019). Most importantly, the innate immune response of larvae is much similar to that of vertebrates, such as identical phagocytic mechanisms, cell surface receptors, and cell signaling pathways (Cutuli et al., 2019; Tsai et al., 2020). In addition, preexposure to components of microbial cells, antifungals, or physical stress, primes larval immunity to defend against subsequent infection (Huang X.W. et al., 2020; Sheehan et al., 2020). Thus, G. mellonella presents highly functional similarities with aspects of the vertebrate innate immune memory (Netea et al., 2016). Furthermore, many human pathogenic microbes display comparable virulence in G. mellonella and mammals (Desbois and Coote, 2012). Therefore, G. mellonella is a feasible and straightforward alternative model for mammals. Recently, several studies have employed G. mellonella larvae as an animal model to screen nanomaterials (Moya-Anderico et al., 2021b). Although Eskin and his colleagues have reported the toxicity of $\mathrm{ZnO}-\mathrm{NPs}$ in larval hemocyte counts, there is no report on the antifungal activity of ZnO-NPs in G. mellonella larvae (Eskin et al., 2019). Herein, this study aimed to evaluate the antifungal activity of $\mathrm{ZnO}-\mathrm{NPs}$ by utilizing $G$. mellonella larva as an in vivo animal model and clarify the potential mechanism.

\section{MATERIALS AND METHODS}

\section{Preparation of Zinc Oxide Nanoparticle Suspensions}

Zinc oxide nanoparticles were purchased from SigmaAldrich (St. Louis, MO, United States). The particles were dispersed in sterile phosphate-buffered saline (PBS, Thermo Scientific, Wilmington, DE, United States) at a concentration of $100 \mathrm{mg} / \mathrm{ml}$ as the stock solution. Then autoclaved stock solution was diluted in PBS to produce the working solutions at different concentrations. All working suspensions were ultrasonically dispersed for $15 \mathrm{~min}$ prior to experiments.

\section{Characterization of Zinc Oxide Nanoparticles}

The surface morphology of $\mathrm{ZnO}$-NPs was observed by scanning electron microscope (SEM; Zeiss Sigma 300, Oberkochen, Germany). The average particle size and shape of ZnO-NPs were further authenticated by a high-resolution transmission electron microscope (HR-TEM; FEI Tecnai G2 F20 S-TWIN, Hillsboro, OR, United States) at an accelerating voltage of $200 \mathrm{kV}$. The zeta potential of $\mathrm{ZnO}-\mathrm{NPs}$ was measured using a Zetasizer Nano ZS (Nano-ZS; Malvern Instruments, Worcestershire, United Kingdom) at $25^{\circ} \mathrm{C}$ and analyzed by Zetasizer software.

\section{Galleria mellonella and Candida albicans}

Galleria mellonella larvae were purchased from Wax Moth Breeding Inc., Tianjin, China. Larvae, weighing around $250 \mathrm{mg}$, were chosen for all experiments. Since food deprivation increases infection susceptibility of larvae (Junqueira, 2012), larvae were incubated without any food at $15^{\circ} \mathrm{C}$ for $24 \mathrm{~h}$ before use. Treated larvae were kept in Petri dishes and maintained in the dark at $37^{\circ} \mathrm{C}$ without any food source.

Candida albicans standard strain SC5314 was purchased from the American Type Culture Collection (ATCC, Manassas, VA, United States) and cultivated in yeast peptone dextrose (YPD) broth (Sangon Biotech, Shanghai, China) under $200 \mathrm{rpm}$ at $37^{\circ} \mathrm{C}$. 


\section{Toxicity of Zinc Oxide Nanoparticles on Galleria mellonella}

Each testing group contains 15 larvae. Before injection, the larvae were sterilized with $75 \%$ ethanol wipe. The larvae were injected with a Hamilton syringe (Sangon Biotech, Shanghai, China) via the last left proleg. Five microliters of $\mathrm{ZnO}-\mathrm{NP}$ suspensions was administrated at concentrations of $0.1,1,10,20,40$, and $80 \mathrm{mg} / \mathrm{ml}$, which are equivalent to the dosages of $2,20,200$, 400,800 , and $1,600 \mathrm{mg} / \mathrm{kg}$, respectively, for a larva weighing $250 \mathrm{mg}$. The same volume of sterilized PBS was injected as the treatment control. After inoculation, the larvae were transferred to Petri dishes and maintained at $37^{\circ} \mathrm{C}$ for 8 days in the dark. The daily observation was performed to record larval mortality. Dead larvae are inert and usually turned black. Every experiment was thrice repeated. The toxicity of $\mathrm{ZnO}-\mathrm{NPs}$ is represented by larval mortality.

\section{Infection Model of Zinc Oxide Nanoparticle-Pretreated Galleria mellonella}

Each testing group contains 15 larvae. The non-toxic doses $(0.2$ and $2 \mathrm{mg} / \mathrm{kg}, 5 \mu \mathrm{l}$ ) of $\mathrm{ZnO}-\mathrm{NPs}$ were chosen for prophylactic injection to ascertain the effects of $\mathrm{ZnO}-\mathrm{NPs}$ on controlling C. albicans sublethal infection in G. mellonella larvae. Larvae pretreated with amphotericin B ( $4 \mathrm{mg} / \mathrm{kg}, 5 \mu \mathrm{l})$ and sterilized PBS were used as the positive and negative controls, respectively. Two hours later, the insects were challenged with a 70\% lethal dose (LD70) of C. albicans as described previously (Huang X. et al., 2020, Huang X.W. et al., 2020). In brief, each pretreated larva was inoculated with $5 \mu \mathrm{l}$ of $C$. albicans suspensions containing $1 \times 10^{5}$ colony-forming unit (CFU) yeast cells via the last right proleg. After inoculation, larvae were transferred to Petri dishes and maintained at $37^{\circ} \mathrm{C}$ in the dark for an indicated time. Daily observation was conducted for recording larval mortality. The experiment was thrice repeated.

\section{Hemolymph Collection}

Larvae were swabbed with $75 \%$ ethanol and positioned on a lid of Perish dish. The hemolymph was obtained by a syringe in the larval abdomen. Hemolymph that emerged from each larva was separately transferred into a microtube containing icecold insect physiological saline (IPS: $150 \mathrm{mM} \mathrm{NaCl,} 5 \mathrm{mM} \mathrm{KCl}$, $0.1 \mathrm{M}$ Tris- $\mathrm{HCl}, 10 \mathrm{mM}$ EDTA, $30 \mathrm{mM}$ sodium citrate, $\mathrm{pH}$ 6.9) (Procell, Wuhan, China) and $0.002 \%$ phenylthiourea (PTU; Leyan, Shanghai, China).

For PO activity assay, hemolymph was collected in the absence of IPS and PTU.

\section{Histopathological Analysis and Determination of Fungal Burden in Galleria mellonella}

To evaluate the fungal morphology, distribution, and burden in $\mathrm{ZnO}$-NP-pretreated larvae, the histopathological analyses of larvae were performed according to a previously described method (Spadari et al., 2019). Briefly, 48 h after infection,
15 larvae in each group were injected with $100 \mu \mathrm{l}$ of $10 \%$ neutral-buffered formalin via the last left proleg and then fixed in formalin at $4^{\circ} \mathrm{C}$ overnight. After that, the whole larvae were sagittally dissected into two halves and fixed for another $48 \mathrm{~h}$ at $4^{\circ} \mathrm{C}$. Next, samples were dehydrated with gradient ethanol, cleared with xylene, infiltrated with paraffin, embedded in paraffin, and subsequently sectioned at a thickness of $6 \mu \mathrm{m}$. The sections were stained with hematoxylin and eosin (H\&E) and examined using a BX63 optical microscope (OLYMPUS, Tokyo, Japan).

The fungal burden in the hemolymph of the infected larvae was determined after inoculation with C. albicans for 3 and $9 \mathrm{~h}$. Hemolymph was obtained from 15 replicates per group and was 10 -fold diluted in PBS. Then, $100 \mu \mathrm{l}$ of diluted hemolymph was spread to YPD solid medium containing $1 \%$ chloramphenicol and gentamicin. The agar plates were incubated at $37^{\circ} \mathrm{C}$ for $24 \mathrm{~h}$. Colony counts were scored for measuring the fungal load.

\section{Determination of Direct Antifungal Effects of Zinc Oxide Nanoparticles Against Candida albicans}

The direct antifungal activity of $\mathrm{ZnO}-\mathrm{NPs}$ against $C$. albicans was confirmed by determining its minimum inhibitory concentration (MIC) using the broth microdilution methods following the guidance given by the Clinical and Laboratory Standards Institute (National Committee for Clinical Laboratory Standards, 2002). Briefly, $80 \mu \mathrm{l}$ of YPD broth with various concentrations of $\mathrm{ZnO}$ NPs was added to each well of a 96-well plate, and then $20 \mu \mathrm{l}$ of yeast suspension containing $1 \times 10^{5} \mathrm{CFU}$ of $C$. albicans was added into each well. C. albicans incubated with amphotericin B $(0.5 \mu \mathrm{g} / \mathrm{ml})$ was used as the positive control. Candida albicans incubated in the absence of $\mathrm{ZnO}-\mathrm{NPs}$ and amphotericin $\mathrm{B}$ was used as the negative control. After incubation at $37^{\circ} \mathrm{C}$ for $24 \mathrm{~h}$, the growth of $C$. albicans was assessed by measuring optical density at $600 \mathrm{~mm}$. The MIC was defined as the lowest concentration of $\mathrm{ZnO}-\mathrm{NPs}$ at which no visible microbial growth was observed.

In addition, microbiological plating was conducted to evaluate the antifungal effects of ZnO-NPs. Briefly, $100 \mu \mathrm{l}$ of PBS solution containing 1,10 , and $100 \mu \mathrm{g}$ of $\mathrm{ZnO}-\mathrm{NPs}$ was evenly spread on YPD plates containing $1 \%$ chloramphenicol and gentamicin. The same volume of PBS was coated as a control. Subsequently, $50 \mu \mathrm{l}$ of yeast suspension with $60 \mathrm{CFU}$ of $C$. albicans was applied to the coated plates followed by incubation at $37^{\circ} \mathrm{C}$ for $24 \mathrm{~h}$. Colony counts and diameter were recorded for assessing the growth of C. albicans, indicating the antifungal activity of $\mathrm{ZnO}-\mathrm{NPs}$.

\section{Quantification and Morphological Observation of Galleria mellonella Hemocytes}

For total hemocyte quantification and morphological observation, the hemolymph was collected from larva at the indicated time points after $\mathrm{ZnO}-\mathrm{NP}$ pretreatment or C. albicans infection. Next, hemolymph from each larva was 10-fold diluted with ice-cold IPS containing $0.002 \%$ PTU in a 12 -well plate. Twenty microliters of the diluted hemolymph was used for hemocyte counting immediately by an automated cell counter 
(Countstar, Shanghai, China). The remaining hemolymph was used for morphological observation using a BX51 optical microscope (Olympus, Tokyo, Japan).

\section{Scanning Electron Microscopy of Hemocytes}

The microscopic morphology of hemocytes was assessed by SEM according to the method described previously (Kim et al., 2021). The $\mathrm{ZnO}-\mathrm{NP}$-pretreated larvae were challenged with C. albicans for $9 \mathrm{~h}$. Then, hemolymph from 15 larvae in the same group was pooled into a $15-\mathrm{ml}$ centrifuge tube containing IPS and $0.002 \%$ PTU. Hemocytes were sedimented by centrifugation for $200 \times g$ at $4^{\circ} \mathrm{C}$, following by washing thrice with PBS. Next, the samples were resuspended and fixed with $2.5 \%$ glutaraldehyde solution (Leagene Biotechnology, Beijing, China) for $24 \mathrm{~h}$ at $4^{\circ} \mathrm{C}$. Then samples were dehydrated with increasing concentrations of ethanol $(30,50,70,80,90$, and $100 \%)$ and placed on an aluminum dish overnight to dry at room temperature. Subsequently, the samples were coated with gold and observed by SEM (Hitachi S-3000N, Tokyo, Japan).

\section{Phagocytosis Assay of Hemocyte}

The phagocytosis by hemocytes was performed in vivo according to previous studies (Feldman et al., 2012; Wu et al., 2016). In brief, 15 larvae per group were inoculated with $5 \mu \mathrm{l}$ of PBS or $\mathrm{ZnO}-\mathrm{NP}$ suspensions $(2 \mathrm{mg} / \mathrm{kg})$ via the last left proleg. After incubation at $37^{\circ} \mathrm{C}$ for $2 \mathrm{~h}$, each larva was challenged with fluorescein isothiocyanate isomer I (FITC; Solarbio, Beijing, China)-labeled C. albicans $\left(1 \times 10^{5}\right.$ cells, $\left.5 \mu \mathrm{l}\right)$ and incubated at $37^{\circ} \mathrm{C}$ for $3 \mathrm{~h}$ in dark. For preparing FITC-labeled C. albicans, C. albicans suspensions $\left(1 \times 10^{9}\right.$ cells $\left./ \mathrm{ml}\right)$ were incubated with $0.1 \mathrm{mg} / \mathrm{ml}$ in a shaking incubator in the dark for $1 \mathrm{~h}$ and washed with PBS three times.

Subsequently, $50 \mu \mathrm{l}$ of hemolymph was obtained from each larva and diluted in a 12-well plate containing $950 \mu \mathrm{l}$ of IPS and $0.002 \%$ PTU. The plate was gently shaken followed by standing for $15 \mathrm{~min}$ at room temperature. The phagocytosis of C. albicans by hemocytes was visualized using an IX73 fluorescence microscope (Olympus, Tokyo, Japan).

\section{Phenoloxidase Activity Assay}

Phenoloxidase (PO)-catalyzed melanization is a crucial innate defense response in G. mellonella larvae, displaying similarities to the complement system in vertebrates (Sheehan et al., 2020). The phenoloxidase activity in the hemolymph of larvae was measured at 2, 5, 8, and $11 \mathrm{~h}$ after dosing PBS or ZnO-NPs $(2 \mathrm{mg} / \mathrm{kg})$. Moreover, larvae receiving $\mathrm{ZnO}-\mathrm{NP}$ pretreatment and C. albicans infection $\left(1 \times 10^{5}\right.$ cells per larva) were used for phenoloxidase activity detection at 3, 6, and $9 \mathrm{~h}$ after infection. The phenoloxidase activity assay was conducted according to a previous report (Thomaz et al., 2020). Briefly, $2 \mu$ l of hemolymph sample was diluted in $18 \mu \mathrm{l}$ of Tris buffer saline containing $5 \mathrm{mM} \mathrm{CaCl}_{2}$, followed by dilution with $180 \mu \mathrm{l}$ of $2 \mathrm{mM} \mathrm{L-DOPA}$ (Solarbio, Beijing, China) in $50 \mathrm{mM}$ sodium phosphate at $\mathrm{pH}$ 6.5. The enzyme activity was quantified by reading the absorbance at
$490 \mathrm{~nm}$ over $90 \mathrm{~min}$ at 30 -min intervals using a microplate reader (Bio-Rad, Hercules, CA, United States).

\section{Statistical Analysis}

Fifteen larvae were used per group in one experiment, and each experiment was repeated in triplicate. Data were analyzed using the SPSS software 15.0 (SPSS Inc., Chicago, IL, United States). The survival curves of $G$. mellonella were plotted and examined using the Kaplan-Meier method. The differences in the survival curves were determined using the log-rank test. The hemocyte density, colony counts, and phenoloxidase activity level were shown as mean \pm standard deviation (SD). The differences in the mean values between groups were assessed using Student's $t$-test or one-way analysis of variance (ANOVA) methods. A pvalue less than 0.05 in all replicate experiments was considered statistically significant.

\section{RESULTS}

\section{Characterization of Zinc Oxide Nanoparticles}

Scanning electron microscope and HR-TEM were used to characterize the morphology of $\mathrm{ZnO}-\mathrm{NPs}$. As shown in Figures 1A,B, ZnO-NPs are nanorod shaped with an average size of $100 \mathrm{~nm} \times 15 \mathrm{~nm}$. Zeta potential, one of the fundamental parameters to evaluate the physical stability of nanosuspension, reflects the electric potential difference at the particle-liquid interface (Dananjaya et al., 2020). The zeta potential of ZnO-NPs was calculated as $+25.9 \mathrm{mV}$ (Figure 1C), inferring that $\mathrm{ZnO}-\mathrm{NPs}$ exhibited good stability in water.

\section{Low Doses of Zinc Oxide Nanoparticles Were Safe to the Galleria mellonella Larvae}

The in vivo toxicity of $\mathrm{ZnO}-\mathrm{NPs}$ was assessed by utilizing G. mellonella larvae. We observed that the $\mathrm{ZnO}-\mathrm{NP}$ treatment affected larval survival in a dose-dependent manner (Figure 2A). Specifically, the evident toxicity of $\mathrm{ZnO}-\mathrm{NPs}$ on larvae was noticed at concentrations greater than $400 \mathrm{mg} / \mathrm{kg}$. The LD50 and maximum LD (LD99) values of $\mathrm{ZnO}-\mathrm{NPs}$ were 400 and $800 \mathrm{mg} / \mathrm{kg}$, respectively. In addition, all larvae died at the second day after $\mathrm{ZnO}-\mathrm{NPs}$ treatment at $1,600 \mathrm{mg} / \mathrm{kg}$. As the injected dose of $\mathrm{ZnO}-\mathrm{NPs}$ decreased, the larval survival rate increased. As illustrated in Figure 2A, treatments with 20 and $2 \mathrm{mg} / \mathrm{kg}$ of $\mathrm{ZnO}-\mathrm{NPs}$ did not show any significant difference in the larval survival compared with PBS-treated control. Especially, no larva died after receiving $2 \mathrm{mg} / \mathrm{kg}$ of $\mathrm{ZnO}-\mathrm{NP}$ treatment. These results indicated that low doses of $\mathrm{ZnO}-\mathrm{NPs}$ were non-toxic to larvae and could be administrated in animal model research.

\section{Zinc Oxide Nanoparticle Pretreatment Improved the Survival of Candida albicans-Infected Larvae}

The efficacy of $\mathrm{ZnO}$-NPs defending against fungal infection was investigated in G. mellonella larvae. According to our previous 

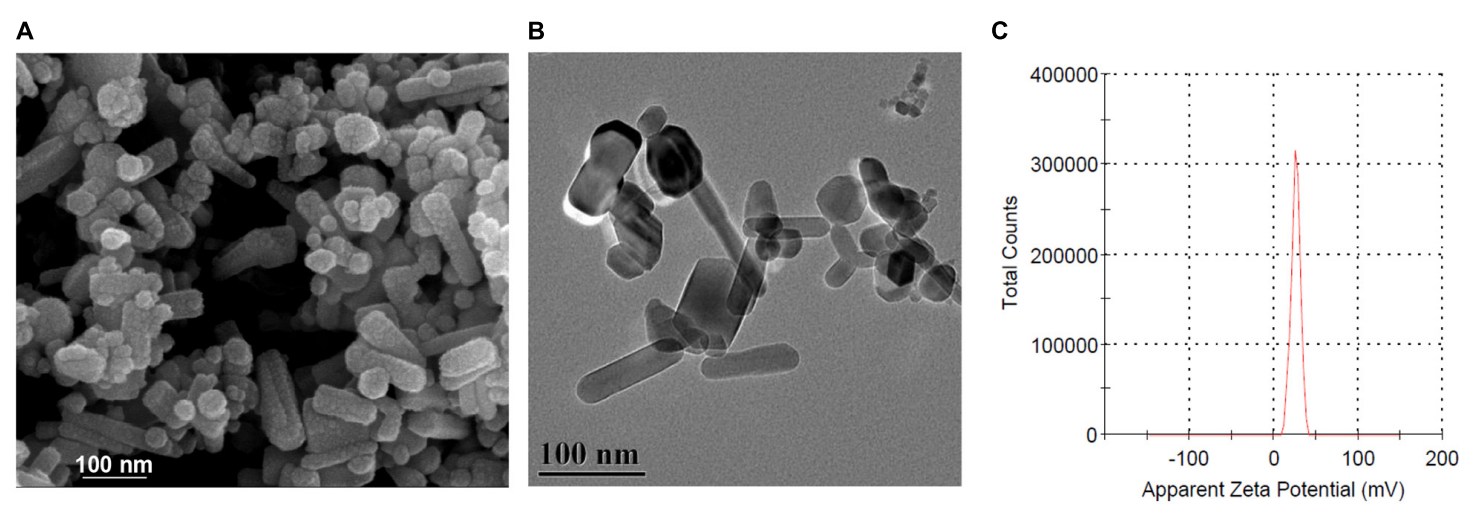

FIGURE 1 | Characterization of zinc oxide nanoparticles (ZnO-NPs). (A) Scanning electron microscopy (SEM) and (B) high-resolution transmission electron microscope (HRTEM) showing the morphology of ZnO-NPs. Scale bar, $100 \mu \mathrm{m}$. (C) Zeta potential of ZnO-NPs.

research (Netea et al., 2016) and the in vivo toxicity results of $\mathrm{ZnO}-\mathrm{NPs}$ on G. mellonella larvae (Figure 2A), $\mathrm{ZnO}-\mathrm{NPs}$ at a non-toxic concentration of $2 \mathrm{mg} / \mathrm{kg}$ were applied for larval pretreatment in the following studies. Also, a relatively lower concentration $(0.2 \mathrm{mg} / \mathrm{kg})$ was performed to examine the dosedependent bio-effects of $\mathrm{ZnO}-\mathrm{NPs}$. As shown in Figure 2B, the pretreated larvae were challenged with a 70\% lethal dose (LD70) of C. albicans instead of a full lethal dose. After 8 days, $67 \%$ of the PBS-pretreated control larvae and $27 \%$ of amphotericin B-pretreated (4 mg/kg) larvae died of C. albicans infection. Meanwhile, after challenging with $C$. albicans, the ZnO-NPpretreated larvae displayed significant viability compared with PBS-pretreated group. ZnO-NP pretreatment at a dose of $0.2 \mathrm{mg} / \mathrm{kg}$ reduced the larval mortality by $13 \%$ compared with PBS pretreatment $(p>0.05)$. All the infected larvae survived under pretreatment with $\mathrm{ZnO}-\mathrm{NPs}$ at a dose of $2 \mathrm{mg} / \mathrm{kg}$. These results indicated that $\mathrm{ZnO}-\mathrm{NPs}$ were efficient in combating C. albicans at non-toxic doses.

\section{Zinc Oxide Nanoparticle Pretreatment Decreased the Load of Candida albicans in Larvae}

The fungal morphology, distribution, and burden in larval tissue were studied by histopathological analysis $48 \mathrm{~h}$ after being challenged with $C$. albicans. The strain cells on the H\&E staining section were easily distinguished as brown in bright field optics. Figures $\mathbf{3 a}-\mathbf{j}$ showed that $C$. albicans was in yeast morphological form and incorporated in melanized nodules among the tissues. In the $\mathrm{ZnO}-\mathrm{NP}$-pretreated larvae, the strain cells developed smaller nodules localized in the subcuticular area (Figures $\mathbf{3 a}, \mathbf{b}, \mathbf{g}, \mathbf{h}$ ) and fat body (Figures $\mathbf{3 c}, \mathbf{d}, \mathbf{i}, \mathbf{j}$ ) compared with those in the PBS-pretreated controls. Some fungal elements reached the intestinal wall (Figures $\mathbf{3 e}, \mathbf{f}$ ) in PBS-pretreated larvae, while no fungus was detected in the intestines of $\mathrm{ZnO}$ NP-pretreated larvae (Figures $\mathbf{3 k} \mathbf{k} \mathbf{l}$ ). Hence, the administration of $\mathrm{ZnO}$-NPs effectively reduced $C$. albicans dissemination in the larval tissue.

Hemolymph collected from infected larvae pretreated with $\mathrm{ZnO}-\mathrm{NPs}$ or PBS was spread on YPD plates to quantify the fungal burden. As shown in Figure $3 \mathrm{~m}, 3 \mathrm{~h}$ after C. albicans infection, no significant difference in fungal load was observed between $\mathrm{ZnO}-\mathrm{NPs}$ and PBS-pretreated larvae $(9.33 \pm 0.88$ vs. $10.33 \pm 0.88 \mathrm{CFU}, p=0.46)$. Interestingly, $9 \mathrm{~h}$ after infection, the fungal load statistically decreased in the $\mathrm{ZnO}-\mathrm{NP}$-pretreated larvae but increased in the PBS control group $(7.33 \pm 1.33$ vs. $63.00 \pm 4.36 \mathrm{CFU}, p=0.0003$ ), which demonstrated that $\mathrm{ZnO}-\mathrm{NPs}$ contribute to the killing of fungus within larvae. Particularly, $9 \mathrm{~h}$ rather than $3 \mathrm{~h}$ of inoculation of $\mathrm{ZnO}-\mathrm{NPs}$ triggered a noticeable antifungal effect. Accordingly, it can be inferred that it takes a longer time for $\mathrm{ZnO}-\mathrm{NPs}$ to exert in vivo antifungal activity, indicating that this antifungal effect might not be a direct effect.

\section{Zinc Oxide Nanoparticles Possessed No Direct Antifungal Capability Against Candida albicans in vitro}

To figure out whether the saving of infected larvae by $\mathrm{ZnO}-\mathrm{NPs}$ was due to the direct antifungal capability of the nanomaterials, in vitro antifungal activity of $\mathrm{ZnO}-\mathrm{NPs}$ against $C$. albicans was first evaluated by liquid broth assay. C. albicans was cultured in YPD broth containing different concentrations of $\mathrm{ZnO}-\mathrm{NPs}$ $(0-1,000 \mu \mathrm{g} / \mathrm{ml})$ at $37^{\circ} \mathrm{C}$ for $24 \mathrm{~h}$. As shown in Figure $4 \mathrm{~A}$, $0.5 \mu \mathrm{g} / \mathrm{ml}$ of amphotericin B was utilized as positive control. However, no significant growth inhibition was observed in the $\mathrm{ZnO}-\mathrm{NP}$-treated groups. It could be speculated that $\mathrm{ZnO}$ NPs did not inhibit the growth of C. albicans directly at the tested concentrations.

Moreover, the growth of C. albicans was assessed on YPD plates coated with 1,10 , and $100 \mu \mathrm{g}$ of $\mathrm{ZnO}$-NPs on the agar surfaces. As shown in Figure 4B, there was no significant difference in CFU of C. albicans between ZnO-NPs and PBScoated plates. Meanwhile, we did not observe any apparent discrepancy in the colony diameter of all groups, indicating no growth inhibition. These results confirmed that $\mathrm{ZnO}-\mathrm{NPs}$ did not possess fungicidal effects at the tested concentrations. Hence, we concluded that the protective role of $\mathrm{ZnO}-\mathrm{NPs}$ in G. mellonella against C. albicans infection did not depend on their fungicidal capability. 

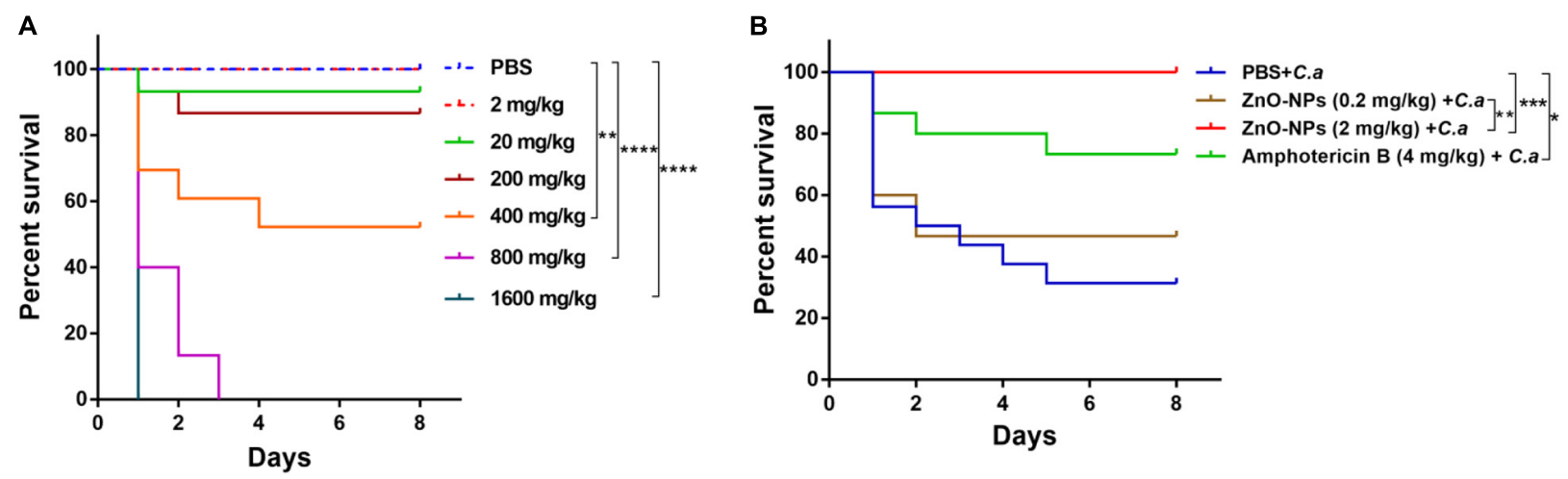

FIGURE 2 | The toxicity of ZnO-NPs on Galleria mellonella and the efficacy of ZnO-NPs saving G. mellonella from Candida albicans infection. (A) Survival curves of G. mellonella larvae injected with various concentrations of ZnO-NPs. Larvae were injected with ZnO-NP suspensions at concentrations ranging from 2 to $1,600 \mathrm{mg} / \mathrm{kg}$. Phosphate-buffered saline (PBS) was injected as the control. (B) Survival curves of G. mellonella receiving ZnO-NP pretreatment and C. albicans challenge. Larvae were pretreated with ZnO-NPs at non-toxic concentrations ( 0.2 and $2 \mathrm{mg} / \mathrm{kg})$. Pretreatment with amphotericin B $(4 \mathrm{mg} / \mathrm{kg}, 5 \mu \mathrm{l})$ and sterilized PBS was used as the positive and negative controls, respectively. Larvae were immunized with $1 \times 10^{5}$ colony-forming units (CFU) of C. albicans $2 \mathrm{~h}$ postinoculation. Fifteen larvae were used per group in one experiment. The experiments were repeated in triplicate independently. Differences were determined by using the log-rank test. ${ }^{*} p<0.05 ;{ }^{* *} p<0.01 ;{ }^{* \star *} p<0.001 ;{ }^{* * *} p<0.0001$.

\section{Zinc Oxide Nanoparticles Increased the Larval Hemocyte Density}

We continued to investigate the ability of $\mathrm{ZnO}-\mathrm{NPs}$ in enhancing the innate immune responses of G. mellonella. We quantified the major parameters of the larval immune response, including hemocyte density and aggregation, phagocytosis ability of hemocyte, and phenoloxidase activity of hemolymph.

First, the hemocyte density in ZnO-NPs-treated G. mellonella was measured in the absence of $C$. albicans infection. As indicated in Figure 5A (left), the increased hemocyte density in the larvae $2 \mathrm{~h}$ post-ZnO-NP treatment, with a recording of $19.73 \pm 1.95 \times 10^{3}$ cells $/ \mu$ l, significantly differed from those of $9.52 \pm 0.81 \times 10^{3}$ cells $/ \mu l$ in the PBS-treated larvae $(p=0.0091)$. Second, we measured the larval hemocyte density in $\mathrm{ZnO}$ NP-pretreated G. mellonella at the indicated time postinfection of C. albicans (Figure 5A). Following infection for $3 \mathrm{~h}$, the larvae pretreated with $\mathrm{ZnO}$-NPs presented increased hemocyte density $\left(14.07 \pm 1.62 \times 10^{3}\right.$ cells/ $\left.\mu l\right)$ compared with the larvae pretreated with PBS as control $\left(6.04 \pm 1.03 \times 10^{3}\right.$ cells $/ \mu \mathrm{l}, p=0.014)$. Furthermore, $9 \mathrm{~h}$ postinfection, there was no significant difference in hemocyte density between the two groups $\left(5.11 \pm 0.56 \times 10^{3}\right.$ cells $/ \mu \mathrm{l} v$ s. $5.32 \pm 1.04 \times 10^{3}$ cells $/ \mu \mathrm{l}$, $p=0.87$.

As for the decrease in hemocyte density after $9 \mathrm{~h}$ of infection, it may be related to the consumption of hemocytes in nodulation or encapsulation process (Kavanagh and Reeves, 2004). Therefore, we speculated that $\mathrm{ZnO}-\mathrm{NP}$ treatment increased the hemocyte density of larvae, contributing to defending against $C$. albicans.

\section{Zinc Oxide Nanoparticles Promoted the Aggregation of Larval Hemocytes}

The aggregation of hemocytes in larvae is one of their innate immune responses, which was observed under microscopy in this study. Hemocytes in hemolymph were extracted from the 2 h-pretreated larvae for microscopy. As shown in Figure 5B, hemocytes formed small aggregates in response to the administration of ZnO-NPs. No hemocyte aggregates were found in the control group injected with PBS.

Furthermore, combined with $3 \mathrm{~h}$ exposure to C. albicans, intense aggregation of hemocytes was observed in the larvae pretreated with $\mathrm{ZnO}-\mathrm{NPs}$, presenting as nodule formation, while cells in the control group were still distributed separately (Figure 5B). With the extension of infection time to $9 \mathrm{~h}$, we noticed a reduction in large nodules in the ZnO-NP-pretreated group, in which the aggregation of hemocytes was similar to that of the control group.

Based on the above results, it was concluded that $\mathrm{ZnO}-\mathrm{NPs}$ could increase the hemocyte density and induce the formation of microaggregates, resulting in a rapid immune response to invasive pathogens, which presented as intense aggregation around the pathogen.

\section{Micromorphology Changes of Hemocytes in Galleria mellonella}

Scanning electron microscopy was used for capturing the micromorphology of hemocytes in the ZnO-NP-pretreated larvae with/without $C$. albicans challenge. First, Figure 6A presents the differences in morphology hemocytes collected from the larvae treated with ZnO-NPs or PBS in the absence of infection. Specifically, hemocytes from ZnO-NPs-treated larvae shrunk and had some burring protrusion on the surface, indicating cell activation. Following inoculation with C. albicans for $9 \mathrm{~h}$, the phagocytosis of C. albicans by hemocytes was captured and is shown in Figure 6B. With PBS pretreatment, hemocytes presented irregularly and were partly destroyed by C. albicans. With $\mathrm{ZnO}-\mathrm{NP}$ pretreatment, the phagocytosis showed more favorably, without significant morphological changes in hemocytes. 

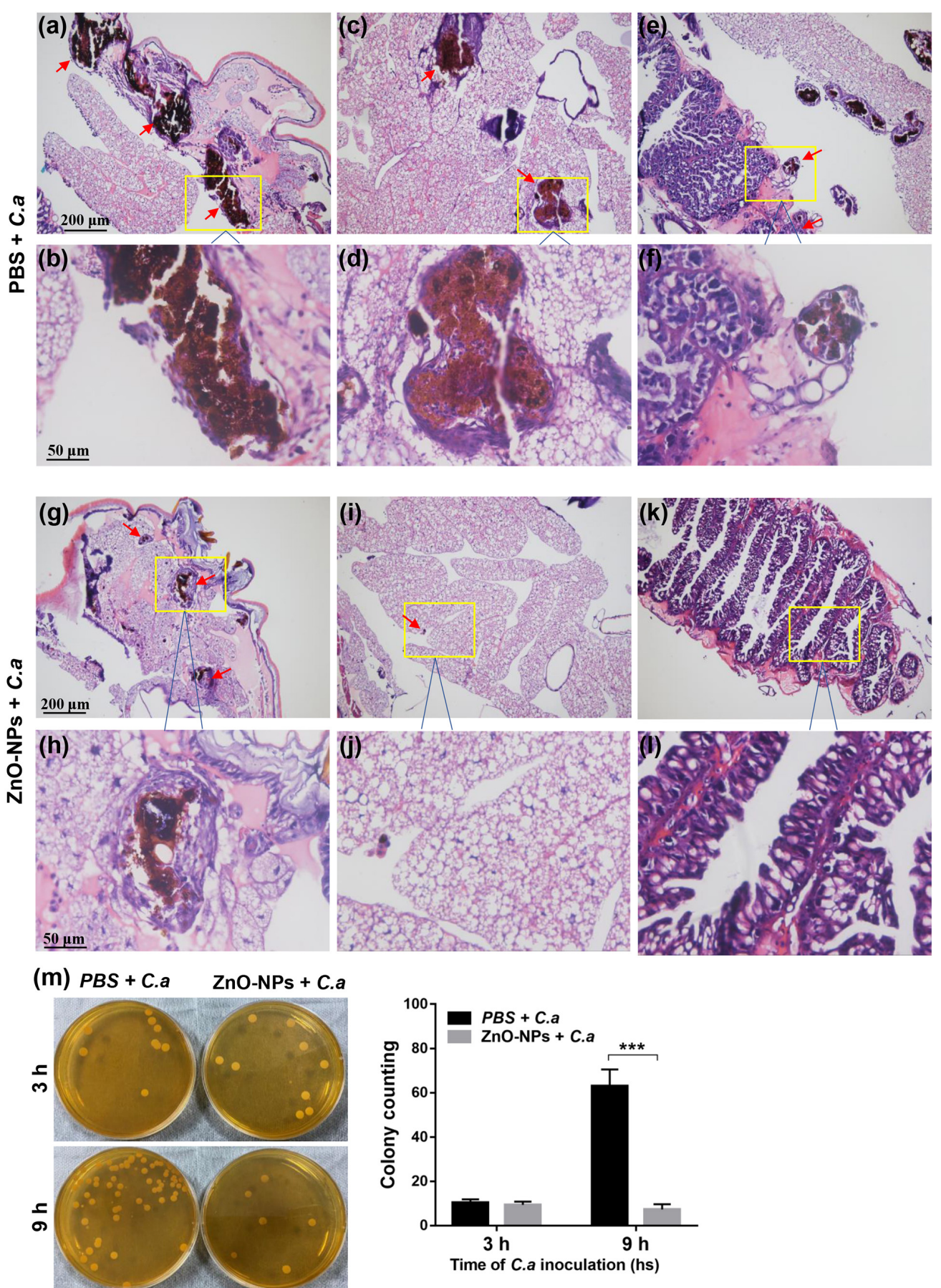

FIGURE 3 | Histopathological analysis and determination of fungal burden in G. mellonella. (a-I) Representative photomicrographs of the fungal morphology, distribution, and burden in G. mellonella on day 2 after ZnO-NP pretreatment and C. albicans inoculation. G. mellonella pretreated with PBS and inoculated with C. albicans was used as the control. Stained sections are shown at $\times 100$ magnification (a,c,e,g,i,k) and $\times 400$ magnification (b,d,f,h,j,l), respectively. C. albicans were in yeast morphological form and incorporated in melanized nodules among tissues. The strain isolates were visualized in subcuticular areas (a,b,g,h), fat bodies (c, d,i,j), and intestinal walls (e,f). Smaller nodules were observed in ZnO-NP-pretreated G. mellonella (g-j) than in PBS-pretreated control group (a-f). (k,l) No fungus was detected in the intestines of ZnO-NP-pretreated G. mellonella. (m) The fungal burden in the hemolymph of PBS or ZnO-NP-pretreated G. mellonella after challenge with C. albicans $\left(1 \times 10^{5} \mathrm{CFU} /\right.$ larva). At 3 and $9 \mathrm{~h}$ postinfection, hemolymph was obtained from 15 replicates per group and was diluted 10 times with PBS. Then $100 \mu \mathrm{l}$ of diluted hemolymph was plated onto yeast peptone dextrose (YPD) solid medium and incubated at $37^{\circ} \mathrm{C}$ for $24 \mathrm{~h}$. Colony counts were measured and shown as mean $\pm \mathrm{SD}$. Differences were determined by using the $t$-test. ${ }^{\star \star \star} p<0.001$. 
A

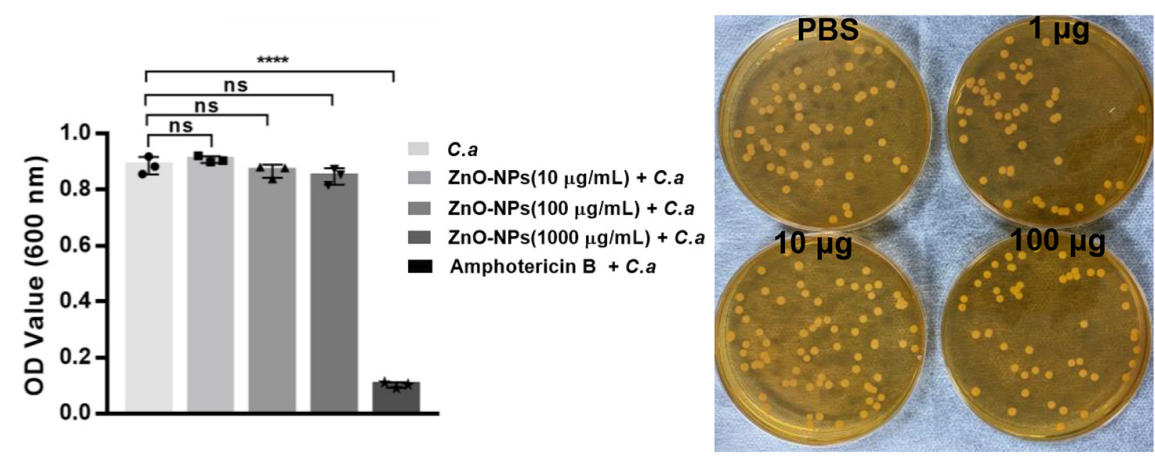

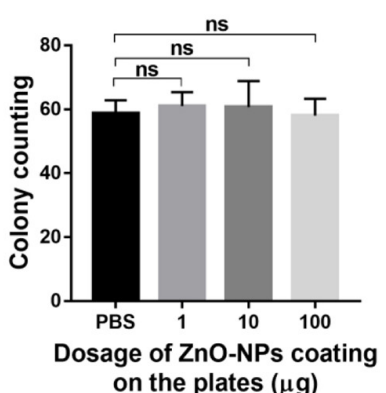

on the plates $(\mu \mathrm{g})$

FIGURE 4 | The direct antifungal capability of ZnO-NPs against C. albicans in vitro. (A) The growth of $C$. albicans in YPD broth containing different concentrations of $\mathrm{ZnO}-\mathrm{NPs}(0-1,000 \mu \mathrm{g} / \mathrm{ml})$ after overnight incubation. C. albicans incubated with amphotericin B $(0.5 \mu \mathrm{g} / \mathrm{ml})$ were used as the positive control. C. albicans incubated in the absence of ZnO-NPs and amphotericin B were used as the negative control. The minimum inhibitory concentration (MIC) value was assessed by measuring absorbance at $600 \mathrm{~nm}$. (B) The growth of $\mathrm{C}$. albicans on YPD plates coated with ZnO-NPs. One hundred microliters of PBS solutions containing 1 , 10 , and $100 \mathrm{\mu g}$ of ZnO-NPs were evenly spread on YPD plates followed by plating with approximately $60 \mathrm{CFU}$ of $\mathrm{C}$. albicans and incubation at $37^{\circ} \mathrm{C}$ for 24 h. The same volume of PBS was coated as a control. Colonies were captured and counted for assessing the fungal growth. Each experiment was repeated in triplicate independently. Data are shown as mean $\pm \mathrm{SD}$. The differences in the mean values between groups were assessed using one-way analysis of variance (ANOVA) method.

\section{Zinc Oxide Nanoparticles Enhanced the Phagocytosis of Larval Hemocytes on Candida albicans}

Furthermore, we utilized fluorescent microscopy to assess the phagocytosis of larval hemocytes against C. albicans. The fungus cells were labeled with FITC and injected into $\mathrm{ZnO}-\mathrm{NP}$ pretreated larvae. As shown in Figure 6C, $3 \mathrm{~h}$ postinfection, hemocytes in the larvae pretreated with $\mathrm{ZnO}$-NPs were more susceptible and aggressive to wrap up C. albicans than cells in the PBS-pretreated larvae.

\section{Zinc Oxide Nanoparticles Activated Phenoloxidase Activity of Larval Hemolymph}

The activation of phenoloxidase in the hemolymph is critical due to the involvement in immune defense and was examined with/without C. albicans infection in the pretreated larvae in the current study. The phenoloxidase enzyme activity was quantified by $\mathrm{OD}_{490}$ value with a microplate reader.

First, larvae were treated with $\mathrm{ZnO}-\mathrm{NPs}$ or PBS only in the absence of infection. As shown in Figure 7A, hemolymph was extracted $2-11 \mathrm{~h}$ after the ZnO-NP injection, while PBS treatment was utilized as the control. At the indicated time points, the phenoloxidase activity was monitored over $90 \mathrm{~min}$ at 15 min intervals. In the ZnO-NP-injected larvae, the phenoloxidase activity remained at a higher level than in the PBS-injected controls (Figure 7A). The phenoloxidase activity peaked $2 \mathrm{~h}$ postinoculation of $\mathrm{ZnO}-\mathrm{NPs}$, presenting 2.57-fold higher activity than that of PBS-inoculated control larvae $(0.654 \pm 0.057$ vs. $0.254 \pm 0.049, p<0.0001)$. We could tell from Figure 7A that phenoloxidase activity showed a trend of decrease according to the prolongation of ZnO-NP treatment from 2 to $11 \mathrm{~h}$. Until $11 \mathrm{~h}$ postinjection, the phenoloxidase enzyme activity was equal in ZnO-NP and PBS-treated larvae $(0.246 \pm 0.077$ vs. $0.213 \pm 0.058$, $p=0.43$; Figure 7A). Thus, according to the results, the administration of $\mathrm{ZnO}-\mathrm{NPs}$ activated the phenoloxidase system. Moreover, the activation of larval phenoloxidase took place in a short time after $\mathrm{ZnO}-\mathrm{NP}$ inoculation and then became attenuated over time lapse.

Second, we detected the phenoloxidase activity levels in ZnO-NPs and PBS-pretreated larvae 3, 6, and $9 \mathrm{~h}$ postchallenge with C. albicans. As shown in Figure $7 \mathrm{~B}, 3 \mathrm{~h}$ postinfection, the larvae pretreated with $\mathrm{ZnO}$-NPs exhibited 2.04-fold higher phenoloxidase activity than the PBS-pretreated controls $(0.627 \pm 0.136$ vs. $0.307 \pm 0.036, p=0.0009)$. The activation of phenoloxidase $2 \mathrm{~h}$ post-inoculation of ZnO-NPs may contribute to the enhanced immune response defending against the subsequent $C$. albicans infection. As the infection time extended to $6 \mathrm{~h}$, the phenoloxidase activity reduced in the ZnO-NP-pretreated larvae $(0.355 \pm 0.154)$ but increased in the PBS-pretreated ones $(0.417 \pm 0.098)$. There was no significant difference in the phenoloxidase activity of the groups at either 6 or $9 \mathrm{~h}$ after infection. The activation of the phenoloxidase system by $\mathrm{ZnO}-\mathrm{NPs}$ in G. mellonella at the early stage probably plays a role in defending against the subsequent infections.

\section{DISCUSSION}

During the last decade, lots of NPs have been reported as novel and sustainable antifungal materials (Staron and Dlugosz, 2021). A previous work has revealed that $\mathrm{ZnO}-\mathrm{NPs}$ have a wide antifungal range and relatively low toxicity (Sharma et al., 2020). However, the data of $\mathrm{ZnO}$-NPs were primarily obtained from in vitro assays. Thus, we attempted to figure out the in vivo toxicity and antifungal activity of ZnO-NPs. Galleria mellonella larvae have been widely used as an animal model to study the pathogenesis of microorganisms and assess the antimicrobial activity of new compounds (Frei et al., 2021). In addition, some 
A

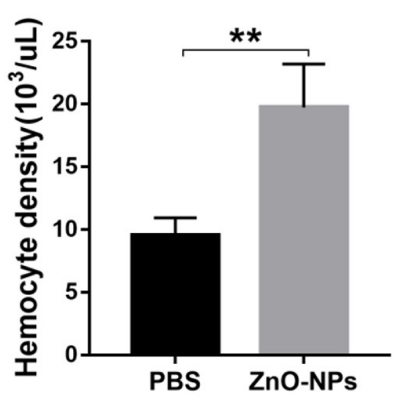

B
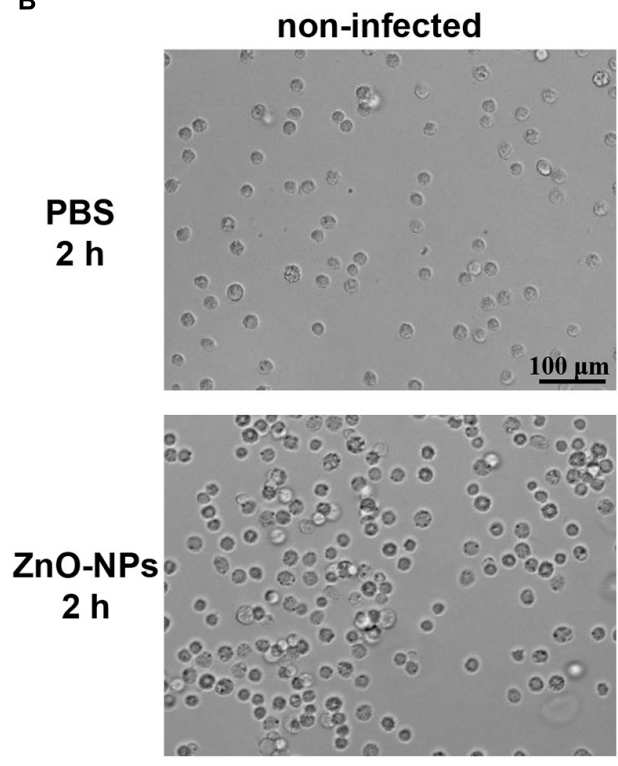

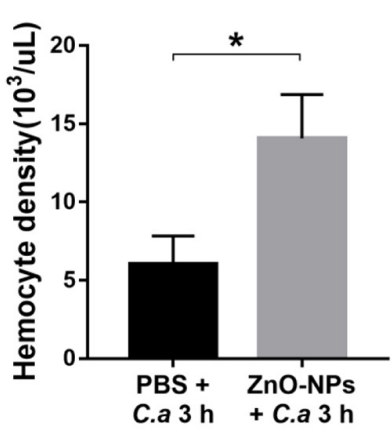

C.a $3 \mathrm{~h}$
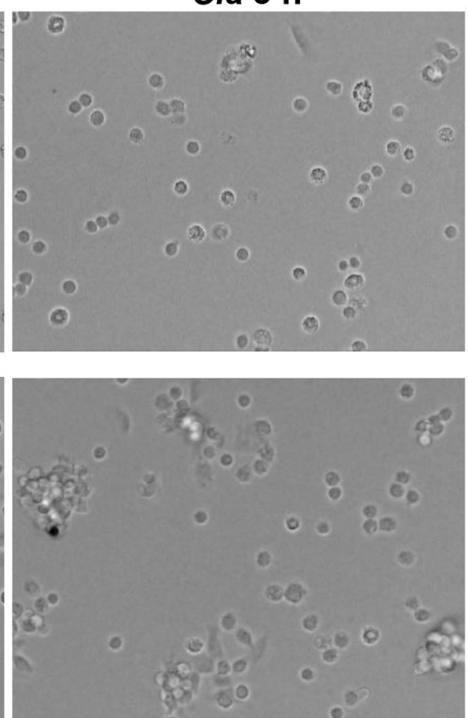

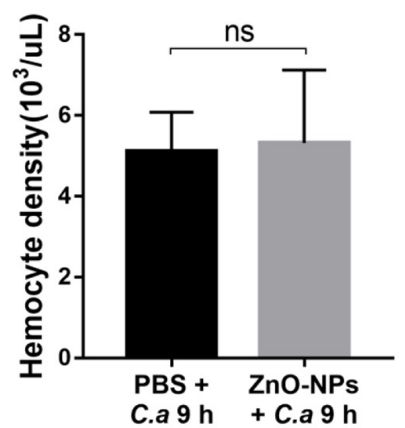

C. 9 h

FIGURE 5 | The density and aggregation of hemocytes in ZnO-NP-pretreated G. mellonella with/without C. albicans challenge. (A) Quantification of hemocytes in ZnO-NP-pretreated G. mellonella with/without C. albicans infection. Differences were determined by using the t-test. * $p<0.05$; ${ }^{* *} p<0.01$. (B) Representative photomicrographs showing the aggregation of hemocytes in ZnO-NP-pretreated G. mellonella with/without $C$. albicans infection. Hemocytes formed small aggregates in response to the administration of $\mathrm{ZnO}-\mathrm{NPs}$ in the absence of $\mathrm{C}$. albicans infection. After 3-h exposure to $\mathrm{C}$. albicans, intense aggregation of hemocytes was observed in ZnO-NP-pretreated G. mellonella. Scale bar, $100 \mu \mathrm{m}$.

research extended the utilization of larvae to study NPs (MoyaAnderico et al., 2021a,b). Given that C. albicans is one of the most common fungal pathogens and has increased resistance to traditional antifungal agents, we established a G. mellonellaC. albicans infection model to elucidate the antifungal capacity of $\mathrm{ZnO}-\mathrm{NPs}$. We herein got an essential finding that $\mathrm{ZnO}-\mathrm{NPs}$ contribute to saving larvae from $C$. albicans infection at low doses without noticeable toxicity. Intriguingly, $\mathrm{ZnO}-\mathrm{NPs}$ protected the larvae against fungal infection by inducing the innate immune responses rather than directly killing the fungus in vivo. An understanding of the mechanisms driving the antimicrobial ability of ZnO-NPs is important for proposing new strategies to eliminate the burden of $C$. albicans and to prevent host death.

A nanoparticle refers to a microscopic particle with a size less than $100 \mathrm{~nm}$ in one or more external dimensions (Lavker et al., 2021). The ZnO-NPs used in this study are nanorod shaped and with an average size of $100 \mathrm{~nm} \times 15 \mathrm{~nm}$. The shape and size of $\mathrm{ZnO}-\mathrm{NPs}$ reported in the literature were diverse, such as spherical shape with a size of 7.41-13 nm (David et al., 2021), crystallite shape with a size of 5-50 nm (Saleh et al., 2021), and rod shape with a size of $88 \mathrm{~nm}$ (Ebadi et al., 2021). The size and shape of NPs play an essential role in determining their toxicity (Sukhanova et al., 2018). For example, NPs larger than $10 \mathrm{~nm}$ are less toxic than NPs in sizes of several nanometers because larger NPs are not easy to enter the nucleus and interact with DNA (Huo et al., 2014). Mesoporous silica NPs in a size of $100 \mathrm{~nm}$ were effectively adsorbed on the surface of the erythrocyte without disturbing the membrane or morphology (Zhao et al., 2011). In addition, in vitro experiment on cultured BEAS-2B cells has shown that needleand plate-shaped NPs produced more potent cytotoxicity than sphere and rod-shaped NPs (Zhao et al., 2013). In the present study, we evaluated the toxicity of ZnO-NPs in G. mellonella larvae by intra-hemocoel injection and determined the LD50 and LD99 values as 400 and $800 \mathrm{mg} / \mathrm{kg}$, respectively. A previous study has reported that the LD50 and LD99 of 70-nm ZnO rodshaped NPs for force-fed larvae were 6.03 and $12.86 \mu \mathrm{g} / 10 \mu \mathrm{l}$, 
A

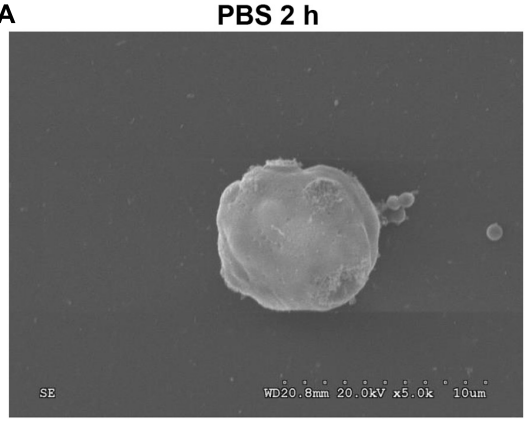

B

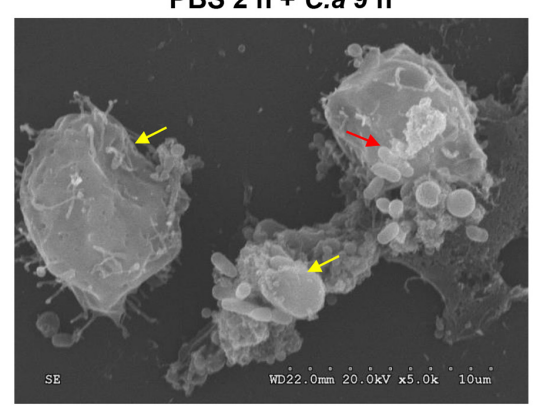

ZnO-NPs 2 h

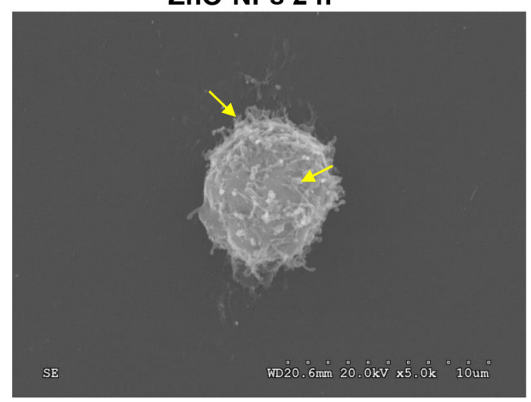

ZnO-NPs $2 \mathrm{~h}+$ C.a $9 \mathrm{~h}$

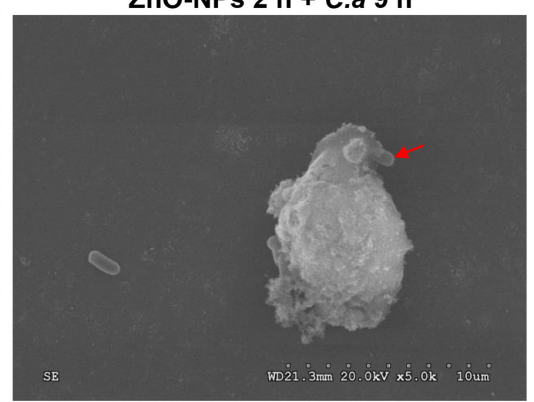

FITC
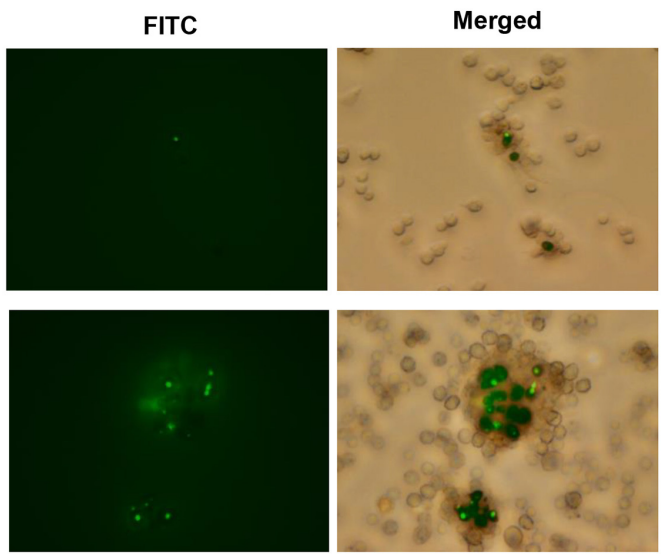

FIGURE 6 | Micromorphology change and phagocytosis of hemocytes in ZnO-NP-pretreated G. mellonella with/without C. albicans challenge. (A,B) Scanning electron microscopy (SEM) presenting micromorphology change of hemocytes collected from ZnO-NP-pretreated G. mellonella with/without $C$. albicans challenge. PBS pretreatment was applied as the control. (A) The yellow arrows indicate shrinking and burring protrusion on the surface of hemocytes exposed to ZnO-NPs without $C$. albicans infection. (B) Following the challenge with $C$. albicans for $9 \mathrm{~h}$, hemocytes presented irregular and partly destroyed by $C$. albicans (yellow arrows) in PBS-pretreated larvae but hemocytes engulfed C. albicans (red arrow) significant morphological changes in ZnO-NP-pretreated larvae. Micromorphology change was shown at $\times 5,000$ magnification. (C) Representative microphotographs showing phagocytosis of fluorescein isothiocyanate isomer (FITC)-labeled C. albicans by hemocytes in G. mellonella. Larvae were pretreated with ZnO-NPs or PBS and then challenged with FITC-labeled C. albicans for $3 \mathrm{~h}$ at $37^{\circ} \mathrm{C}$. Hemolymph was obtained from each larva separately and 10-fold diluted in a 12-well plate for microscope observation. Scale bar, $100 \mu \mathrm{m}$.

respectively, equivalent to 83 and $176 \mathrm{mg} / \mathrm{kg}$ body weight (Eskin et al., 2019). The inconsistent results of the two studies might be due to the difference in the size of $\mathrm{ZnO}-\mathrm{NPs}$, the modes of administration, the larval size and age, and culture conditions.

In this work, $\mathrm{ZnO}-\mathrm{NP}$ pretreatment saved larvae from C. albicans infection at a dose of $2 \mathrm{mg} / \mathrm{kg}$ without apparent toxicity, which is manifested by reducing the larval mortality and the fungal burden in hemolymph and tissue. The mechanisms driving antimicrobial activity of $\mathrm{ZnO}$-NPs have been described in the literature, including generation of ROS, the release of zinc ions, and interaction of $\mathrm{ZnO}-\mathrm{NPs}$ with the surfaces of microorganisms (Behzad et al., 2021; Efthimiou et al., 2021). Particularly, these mechanisms mainly focus on the directly antimicrobial properties of NPs. Intriguingly, we found that $\mathrm{ZnO}-\mathrm{NPs}$ at the tested concentrations did not inhibit the growth of C. albicans. Similarly, reported by Visnapuu et al. (2018), in dark conditions, the $\mathrm{ZnO}$-covered surface did not exhibit a significant antimicrobial effect for C. albicans, while under UVA 

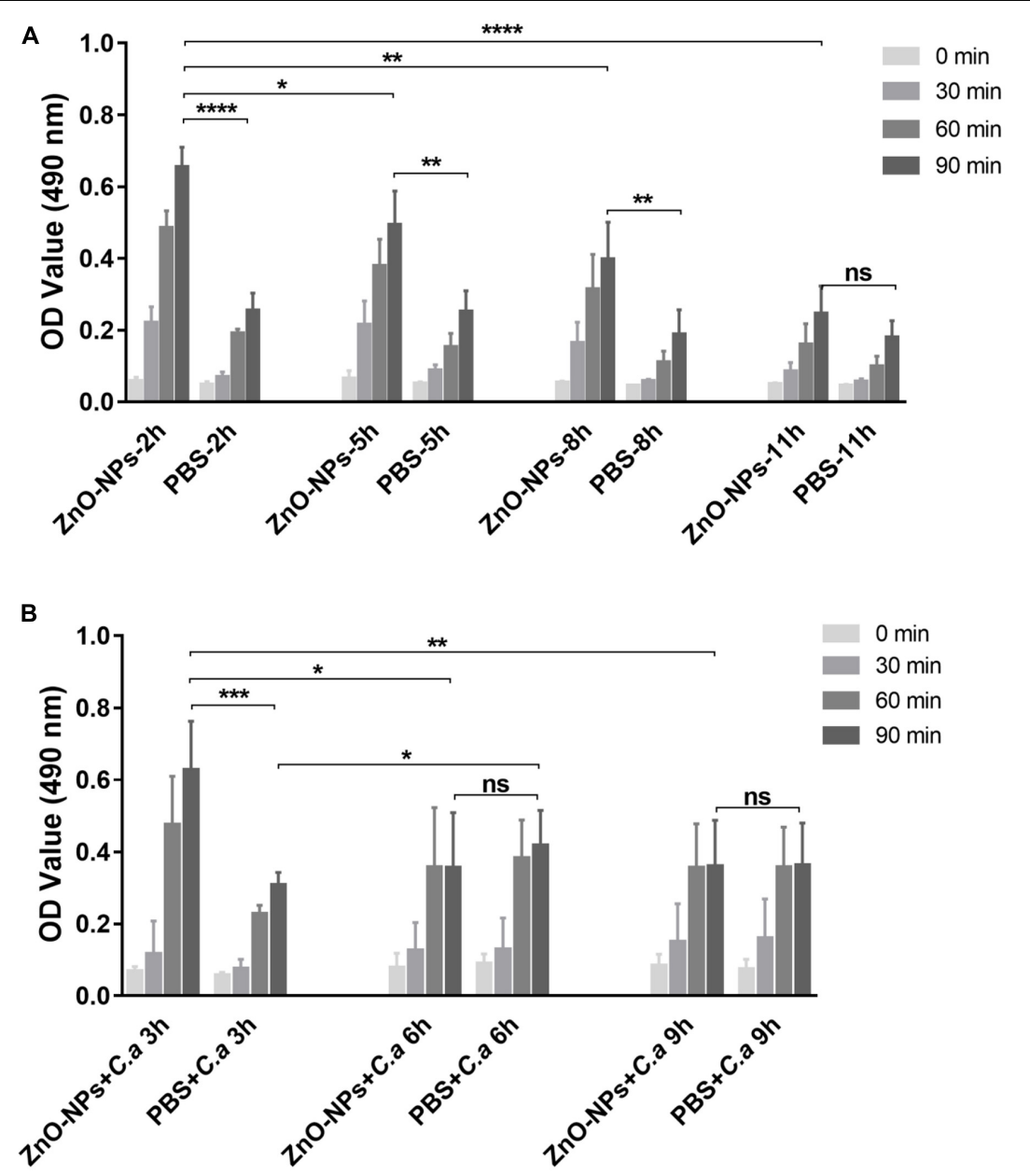

FIGURE 7| Phenoloxidase (PO) activity in the hemolymph of ZnO-NP-pretreated G. mellonella with/without $C$. albicans challenge. (A) The PO activity in the hemolymph of ZnO-NP-treated G. mellonella at indicated time points after the inoculation. The same volume of PBS was injected as the control. (B) The PO activity in the hemolymph of G. mellonella receiving ZnO-NP pretreatment and C. albicans infection. Larvae $2 \mathrm{~h}$ post-ZnO-NPs or PBS treatment were immunized with C. albicans $\left(1 \times 10^{5} \mathrm{CFU}\right.$ cells per larva) and used for detection of PO activity at 3,6 , and $9 \mathrm{~h}$ after challenge. The PO activity was determined using DOPA as a substrate based on melanin formation by measuring absorbance at $490 \mathrm{~nm}$. The enzyme activity was quantified over 90 min at 15-min intervals presented as mean $\pm S D$. The kinetics of the PO activity values were calculated from a representative experiment performed in triplicate. Difference analysis was assessed by using one-way analysis of variance (ANOVA) methods. ${ }^{*} p<0.05 ;{ }^{* \star} p<0.01 ;{ }^{* \star} p<0.001 ;{ }^{* \star * *} p<0.0001$.

illumination, $\mathrm{ZnO}$ was slightly toxic to C. albicans. Moreover, C. albicans was significantly less sensitive to $\mathrm{ZnO}$-covered surface compared with Escherichia coli and Staphylococcus aureus (Visnapuu et al., 2018). The failure of antimicrobial action in our study is probably due to the low level of released $\mathrm{Zn}^{2+}$ ions from ZnO-NPs in the dark and the insensitivity of C. albicans to $\mathrm{Zn}^{2+}$ ions.

Since no direct antifungal activity of $\mathrm{ZnO}-\mathrm{NPs}$ was observed in our study, we assume that the life extension of infected larvae by $\mathrm{ZnO}$-NPs might be mainly attributed to impacting the immune defense in hosts. As we know, the immune system of $G$. mellonella larvae has structural and functional similarities to the innate immune response in mammals, which provides possibilities of utilizing it to study the interaction between hosts and pathogens (Tsai et al., 2020). Considering that hemocytes play a crucial role in cellular immune response as they serve as phagocytic cells, the induced reaction of hemocytes by $\mathrm{ZnO}$-NPs was verified in larvae. Moreover, since phenoloxidase-catalyzed melanization is a key humoral immune response in larvae, the phenoloxidase enzyme activity in larval hemolymph was evaluated. The cellular and humoral immune defense were activated by $\mathrm{ZnO}-\mathrm{NP}$ pretreatment, evidencing increased hemocyte density, aggregation, phagocytosis ability, and phenoloxidase activity. Additionally, in $\mathrm{ZnO}$-NP-treated larvae, the reduced destruction of hemocytes was evident from the SEM micrographs, especially in cells that phagocytized C. albicans. The above evidence indicates that $\mathrm{ZnO}-\mathrm{NP}$ protect G. mellonella against C. albicans infection via immune activation in the host instead of direct fungicidal actions.

The abovementioned mode of $\mathrm{ZnO}-\mathrm{NPs}$ on the immune response was similar to the immune priming effect in G. mellonella. "Immune priming" is an enhanced innate immune 
response that enables invertebrates to withstand potentially lethal infections (Sheehan et al., 2020). This process can be triggered by microbial or components of microbial cells, antifungal drugs, or thermal and physical stress, indicating a generalized antimicrobial response (Sheehan et al., 2020). Our work revealed that the introduction of $\mathrm{ZnO}-\mathrm{NPs}$ into the hemocoel of larvae increased the hemocyte density, even in a short term of $2 \mathrm{~h}$. Hemocytes probably migrated from a sessile population normally present under the larval cuticle rather than de novo synthesis (Kavanagh and Reeves, 2004; Sheehan et al., 2020). Besides, we noticed distinct aggregation of hemocytes and enhanced phagocytosis in response to $\mathrm{ZnO}$ NP injection. These results were in agreement with previous research conducted in vitro that nano- $\mathrm{ZnO}$ films could promote microbial clearance by macrophages and polymorphonuclear leukocytes by facilitating phagocytic efficacy (Rutherford et al., 2021). It has been recently revealed that the phenoloxidase activity of $G$. mellonella was induced after $C$. albicans infection (Sheehan and Kavanagh, 2018). In our study, both ZnO-NPs and PBS-pretreated larvae exhibited increased phenoloxidase activity after C. albicans challenge. However, ZnO-NP-pretreated larvae exhibited a higher peak of phenoloxidase activity at the earlier stage than PBS-pretreated control, implying a stronger immune defense against subsequent infections. Based on our results and preceding studies, it could be concluded that $\mathrm{ZnO}$ NP-induced hemocyte aggregation and phagocytosis as well as phenoloxidase activation in the hemolymph was probably conducive to pathogen clearance quickly and effectively after C. albicans challenge.

\section{CONCLUSION}

In general, we first evaluated the in vivo toxicity of $\mathrm{ZnO}$ NPs on G. mellonella larvae and found the enhanced immune

\section{REFERENCES}

Abo Elgat, W. A. A., Taha, A. S., Bohm, M., Vejmelková, E., Mohamed, W. S., Fares, Y. G. D., et al. (2020). Evaluation of the mechanical, physical, and anti-fungal properties of flax laboratory papersheets with the nanoparticles treatment. Materials (Basel) 13:363. doi: 10.3390/ma13020363

Behzad, F., Sefidgar, E., Samadi, A., Lin, W., Pouladi, I., and Pi, J. (2021). An overview of zinc oxide nanoparticles produced by plant extracts for anti-tuberculosis treatments. Curr. Med. Chem. doi: 10.2174/ 0929867328666210614122109

Bondarenko, O., Juganson, K., Ivask, A., Kasemets, K., Mortimer, M., and Kahru, A. (2013). Toxicity of Ag, $\mathrm{CuO}$ and $\mathrm{ZnO}$ nanoparticles to selected environmentally relevant test organisms and mammalian cells in vitro: a critical review. Arch. Toxicol. 87, 1181-1200.

Burns, K. E., Uhrig, R. F., Jewett, M. E., Bourbon, M. F., and Krupa, K. A. (2021). Characterizing the role of biologically relevant fluid dynamics on silver nanoparticle dependent oxidative stress in adherent and suspension in vitro models. Antioxidants 10:832. doi: 10.3390/antiox10060832

Cutuli, M. A., Petronio Petronio, G., Vergalito, F., Magnifico, I., Pietrangelo, L., Venditti, N., et al. (2019). Galleria mellonella as a consolidated in vivo model hosts: new developments in antibacterial strategies and novel drug testing. Virulence 10, 527-541. doi: 10.1080/21505594.2019.1621649

Dananjaya, S. H. S., Edirisinghe, S. L., Thao, N. T. T., Kumar, R. S., Wijerathna, H. M. S. M., Mudiyanselage, A. Y., et al. (2020). Succinyl chitosan gold response. The relatively low concentrations of $\mathrm{ZnO}-\mathrm{NPs}$ did not display any evident toxicity; however, it primed a protective immune response in the larvae against sublethal fungal infection. These results showed that the low toxicity properties and immunomodulatory potentials of ZnO-NPs make it particularly attractive in medical applications. Especially, the $\mathrm{ZnO}-\mathrm{NP}$-based immunomodulator is a promising tool to defend against fungal infections. Future research is worth delineating the molecular mechanisms of immunomodulatory effects mediated by $\mathrm{ZnO}$ NPs and whether the $\mathrm{ZnO}$-NP-induced immune activation could be extended to other NPs.

\section{DATA AVAILABILITY STATEMENT}

The raw data supporting the conclusions of this article will be made available by the authors, without undue reservation.

\section{AUTHOR CONTRIBUTIONS}

$\mathrm{KZ}$ and $\mathrm{X}-\mathrm{WH}$ conceived the study. M-NX, WP, $\mathrm{H}-\mathrm{XZ}$, and X-MP performed the research. S-QD, Y-MT, and LL analyzed the data. X-WH and M-NX drafted the manuscript. LL and WP edited the manuscript. All authors have read and approved the final manuscript.

\section{FUNDING}

This work was supported by the Outstanding Youth Development Scheme of Nanfang Hospital, Southern Medical University (grant number: 2018J003).

nanocomposite: preparation, characterization, in vitro and in vivo anticandidal activity. Int. J. Biol. Macromol. 165(Pt A), 63-70. doi: 10.1016/j.ijbiomac.2020. 09.126

David, M. E., Ion, R. M., Grigorescu, R. M., Iancu, L., Holban, A. M., Nicoara, A. I., et al. (2021). Hybrid materials based on multi-walled carbon nanotubes and nanoparticles with antimicrobial properties. Nanomaterials 11:1415. doi: 10.3390/nano11061415

Desbois, A. P., and Coote, P. J. (2012). Utility of greater wax moth larva (Galleria mellonella) for evaluating the toxicity and efficacy of new antimicrobial agents. Adv. Appl. Microbiol. 78, 25-53. doi: 10.1016/B978-0-12-394805-2.00 $002-6$

Ebadi, M., Zolfaghari, M. R., Aghaei, S. S., Zargar, M., and Noghabi, K. A. (2021). Desertifilum sp. EAZ03 cell extract as a novel natural source for the biosynthesis of zinc oxide nanoparticles: its antibacterial, anticancer, and antibiofilm characteristics. J. Appl. Microbiol. doi: 10.1111/jam.15177

Efthimiou, I., Kalamaras, G., Papavasileiou, K., Anastasi-Papathanasi, N., Georgiou, Y., Dailianis, S., et al. (2021). ZnO, Ag and $\mathrm{ZnO}-\mathrm{Ag}$ nanoparticles exhibit differential modes of toxic and oxidative action in hemocytes of mussel Mytilus galloprovincialis. Sci. Total Environ. 767:144699. doi: 10.1016/j. scitotenv.2020.144699

Eskin, A., Ozturk, S., and Korukcu, M. (2019). Determination of the acute toxic effects of zinc oxide nanoparticles ( $\mathrm{ZnO} \mathrm{NPs}$ ) in total hemocytes counts of Galleria mellonella (Lepidoptera: Pyralidae) with two different methods. Ecotoxicology 28, 801-808. doi: 10.1007/s10646-019-02078-2 
Feldman, M., Tanabe, S., Howell, A., and Grenier, D. (2012). Cranberry proanthocyanidins inhibit the adherence properties of Candida albicans and cytokine secretion by oral epithelial cells. BMC Complement Altern. Med. 12:6. doi: 10.1186/1472-6882-12-6

Frei, A., King, A. P., Lowe, G. J., Cain, A. K., Short, F. L., Dinh, H., et al. (2021). Nontoxic cobalt(III) schiff base complexes with broad-spectrum antifungal activity. Chemistry 27, 2021-2029. doi: 10.1002/chem.202003545

Gudlaugsson, O., Gillespie, S., Lee, K., Vande Berg, J., Hu, J., Messer, S., et al. (2003). Attributable mortality of nosocomial candidemia, revisited. Clin. Infect. Dis. 37 1172-1177. doi: $10.1086 / 378745$

Hernandez-Melendez, D., Salas-Tellez, E., Zavala-Franco, A., Téllez, G., MéndezAlbores, A., and Vázquez-Durán, A. (2018). Inhibitory effect of flower-shaped zinc oxide nanostructures on the growth and aflatoxin production of a highly toxigenic strain of Aspergillus flavus link. Materials 11:1265. doi: 10.3390/ ma11081265

Huang, X., Xu, M., Pan, W., Wang, M., Wu, X., Dai, S., et al. (2020). Antimicrobial and immunomodulatory responses of photodynamic therapy in Galleria mellonella model. BMC Microbiol. 20:196. doi: 10.1186/s12866-020-01882-9

Huang, X. W., Xu, M. N., Zheng, H. X., Wang, M. L., Li, L., Zeng, K., et al. (2020). Pre-exposure to Candida glabrata protects Galleria mellonella against subsequent lethal fungal infections. Virulence 11, 1674-1684. doi: 10.1080/ 21505594.2020.1848107

Huo, S., Jin, S., Ma, X., Xue, X., Yang, K., Kumar, A., et al. (2014). Ultrasmall gold nanoparticles as carriers for nucleus-based gene therapy due to size-dependent nuclear entry. ACS Nano 8, 5852-5862. doi: 10.1021/nn5008572

Junqueira, J. C. (2012). Galleria mellonella as a model host for human pathogens: recent studies and new perspectives. Virulence 3, 474-476. doi: 10.4161/viru. 22493

Kavanagh, K., and Reeves, E. P. (2004). Exploiting the potential of insects for in vivo pathogenicity testing of microbial pathogens. FEMS Microbiol. Rev. 28 101-112. doi: 10.1016/j.femsre.2003.09.002

Kim, K. J., Sung, W. S., Suh, B. K., Moon, S. K., Choi, J. S., Kim, J. G., et al. (2009). Antifungal activity and mode of action of silver nano-particles on Candida albicans. Biometals 22, 235-242. doi: 10.1007/s10534-008-9159-2

Kim, M., Park, J., and Park, W. (2021). Genomic and phenotypic analyses of multidrug-resistant Acinetobacter baumannii NCCP 16007 isolated from a patient with a urinary tract infection. Virulence 12, 150-164. doi: 10.1080/ 21505594.2020.1867421

Kullberg, B. J., and Arendrup, M. C. (2016). Invasive candidiasis. N. Engl. J. Med. 374, 794-795.

Lavker, R. M., Kaplan, N., McMahon, K. M., Calvert, A. E., Henrich, S. E., Onay, U. V., et al. (2021). Synthetic high-density lipoprotein nanoparticles: good things in small packages. Ocul. Surf. 21, 19-26. doi: 10.1016/j.jtos.2021.03.001

Moya-Anderico, L., Admella, J., and Torrents, E. (2021a). A clearing protocol for Galleria mellonella larvae: visualization of internalized fluorescent nanoparticles. N. Biotechnol. 60, 20-26. doi: 10.1016/j.nbt.2020. 08.002

Moya-Anderico, L., Vukomanovic, M., Cendra, M. D. M., Segura-Feliu, M., Gil, V., Del Río, J. A., et al. (2021b). Utility of Galleria mellonella larvae for evaluating nanoparticle toxicology. Chemosphere 266:129235. doi: 10.1016/j.chemosphere. 2020.129235

National Committee for Clinical Laboratory Standards. (2002). Reference Method for Broth Dilution Antifungal Susceptibility Testing of Yeast. Approved standard M27-A2. Wayne. PA: National Committee for Clinical Laboratory Standards.

Netea, M. G., Joosten, L. A., Latz, E., Mills, K. H., Natoli, G., Stunnenberg, H. G., et al. (2016). Trained immunity: a program of innate immune memory in health and disease. Science 352:aaf1098.

Rutherford, D., Jira, J., Kolarova, K., Matolínová, I., Mičová, J., Remeš, Z., et al. (2021). Growth inhibition of gram-positive and gram-negative bacteria by zinc oxide hedgehog particles. Int. J. Nanomed. 16, 3541-3554. doi: 10.2147/IJN. S300428

Salazar, S. B., Simoes, R. S., Pedro, N. A., Pinheiro, M. J., Carvalho, M. F. N. N., and Mira, N. P. (2020). An Overview on conventional and non-conventional therapeutic approaches for the treatment of candidiasis and underlying resistance mechanisms in clinical strains. J. Fungi. 6:23. doi: 10.3390/ jof6010023
Saleh, F., Kheirandish, F., Hosseini, F., and Yazdian, F. (2021). Evaluation the effect of $\mathrm{ZnO}$ nanoparticle derived Bacillus subtilis on the expression of efflux pump genes (AdeB AdeRS) in Acinetobacter baumannii. J. Environ. Health Sci. Eng. 19, 1133-1141. doi: 10.1007/s40201-021-00679-w

Sharma, R., Garg, R., and Kumari, A. (2020). A review on biogenic synthesis, applications and toxicity aspects of zinc oxide nanoparticles. EXCLI J. 19, 1325-1340. doi: 10.17179/excli2020-2842

Sheehan, G., Farrell, G., and Kavanagh, K. (2020). Immune priming: the secret weapon of the insect world. Virulence 11, 238-246. doi: 10.1080/21505594.2020. 1731137

Sheehan, G., and Kavanagh, K. (2018). Analysis of the early cellular and humoral responses of Galleria mellonella larvae to infection by Candida albicans. Virulence 9, 163-172. doi: 10.1080/21505594.2017.1370174

Spadari, C. C., de Bastiani, F. W. M. D. S., Lopes, L. B., and Ishida, K. (2019). Alginate nanoparticles as non-toxic delivery system for miltefosine in the treatment of candidiasis and cryptococcosis. Int. J. Nanomed. 14, 5187-5199. doi: $10.2147 /$ IJN.S205350

Staron, A., and Dlugosz, O. (2021). Antimicrobial properties of nanoparticles in the context of advantages and potential risks of their use. J. Environ. Sci. Health A Tox. Hazard Subst. Environ. Eng. 56, 680-693. doi: 10.1080/10934529.2021. 1917936

Sukhanova, A., Bozrova, S., Sokolov, P., Berestovoy, M., Karaulov, A., and Nabiev, I. (2018). Dependence of nanoparticle toxicity on their physical and chemical properties. Nanoscale Res. Lett. 13:44.

Sun, Q., Li, J., and Le, T. (2018). Zinc oxide nanoparticle as a novel class of antifungal agents: current advances and future perspectives. J. Agric. Food Chem. 66, 11209-11220. doi: 10.1021/acs.jafc.8b03210

Thomaz, L., Gustavo de Almeida, L., Silva, F. R. O., Cortez, M., Taborda, C. P., and Spira, B. (2020). In vivo activity of silver nanoparticles against Pseudomonas aeruginosa Infection in Galleria mellonella. Front. Microbiol. 11:582107. doi: 10.3389/fmicb.2020.582107

Tsai, C. J., Loh, J. M. S., and Proft, T. (2020). The use of Galleria mellonella (wax moth) as an infection model for group a streptococcus. Methods Mol. Biol. 2136, 279-286. doi: 10.1007/978-1-0716-0467-0_21

Visnapuu, M., Rosenberg, M., Truska, E., Nõmmiste, E., Šutka, A., Kahru, A., et al. (2018). UVA-induced antimicrobial activity of $\mathrm{ZnO} / \mathrm{Ag}$ nanocomposite covered surfaces. Colloids Surf. B Biointerfaces 169, 222-232. doi: 10.1016/j.colsurfb. 2018.05.009

Wu, G., Liu, Y., Ding, Y., and Yi, Y. (2016). Ultrastructural and functional characterization of circulating hemocytes from Galleria mellonella larva: cell types and their role in the innate immunity. Tissue Cell 48, 297-304. doi: 10.1016/j.tice.2016.06.007

Zhao, X., Ng, S., Heng, B. C., Guo, J., Ma, L., Tan, T. T., et al. (2013). Cytotoxicity of hydroxyapatite nanoparticles is shape and cell dependent. Arch. Toxicol. 87, 1037-1052. doi: 10.1007/s00204-012-0827-1

Zhao, Y., Sun, X., Zhang, G., Trewyn, B. G., Slowing, I. I., and Lin, V. S. (2011). Interaction of mesoporous silica nanoparticles with human red blood cell membranes: size and surface effects. ACS Nano 5, 1366-1375.

Conflict of Interest: The authors declare that the research was conducted in the absence of any commercial or financial relationships that could be construed as a potential conflict of interest.

Publisher's Note: All claims expressed in this article are solely those of the authors and do not necessarily represent those of their affiliated organizations, or those of the publisher, the editors and the reviewers. Any product that may be evaluated in this article, or claim that may be made by its manufacturer, is not guaranteed or endorsed by the publisher.

Copyright (c) $2021 \mathrm{Xu}, \mathrm{Li}$, Pan, Zheng, Wang, Peng, Dai, Tang, Zeng and Huang. This is an open-access article distributed under the terms of the Creative Commons Attribution License (CC BY). The use, distribution or reproduction in other forums is permitted, provided the original author $(s)$ and the copyright owner(s) are credited and that the original publication in this journal is cited, in accordance with accepted academic practice. No use, distribution or reproduction is permitted which does not comply with these terms. 